\title{
Development of LiDAR Based Navigation System for Automation of Tree Harvesting Process
}

\author{
Ayumu Tominaga* \\ Graduate School of Computer Science and System Engineering, Kyushu Institute of Technology, 680-4 Kawazu, \\ Iizuka, 820-0044, Fukuoka, Japan ${ }^{\dagger}$ \\ Akihiro Koubara \\ Graduate School of Computer Science and System Engineering, Kyushu Institute of Technology, 680-4 Kawazu, \\ Iizuka, 820-0044, Fukuoka, Japan \\ Ryusuke Fujisawa \\ Graduate School of Computer Science and System Engineering, Kyushu Institute of Technology, 680-4 Kawazu, \\ Iizuka, 820-0044, Fukuoka, Japan \\ Eiji Hayashi \\ Graduate School of Computer Science and System Engineering, Kyushu Institute of Technology, 680-4 Kawazu, \\ Iizuka, 820-0044, Fukuoka, Japan \\ Abbe Mowshowitz \\ Iizuka, 820-0044, Fukuoka, Japan \\ E-mail: tominaga@mmcs.mse.kyutech.ac.jp,koubara.akihiro880@mail.kyutech.ac.jp,fujisawa@ces.kyutech.ac.jp, \\ haya@mse.kyutech.ac.jp
}

\begin{abstract}
This study focuses on an autonomous moving system for the automation of the harvesting process by high-performance machines in the forestry. Many fatal accidents occur due to the harvesting process. In this research, a navigation system has been developed to enable autonomous travel between accumulation sites and trees to be harvested to improve productivity and safety. A 3D map is generated by LiDAR observation, and harvester moves autonomously towards the tree as specified by the operator. A test of the harvesting process was performed in an experimental environment. The evaluation focused on the required time of the autonomous movement in the process. The effectiveness of the system was confirmed in operations such as row thinning by the results.
\end{abstract}

Keywords: Field Robot, Forest Industry, Harvesting, Autonomous Moving

\section{Introduction}

Forestry is an industry that nurtures and economically utilizes forest. The safety of forestry work has become an issue, and the development and proliferation of highperformance forestry machinery has been increasing. In the future, it is necessary to introduce robotics technology to further improve safety and reduce labor burdens by utilizing the mobility applied to the forest environment that has been developed so far.

In this study, we developed a navigation system in the forest environment that is aimed to be operational for the harvesting process. The management style of Japanese forestry is wide-area, small-scale afforestation. 


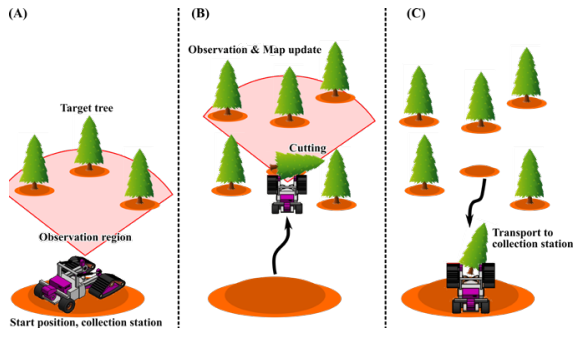

Fig. 1. The scenario of automatic harvesting

Conventional manned machines require long-term, and continuously tree felling and transporting of felled tree, which places a large burden on the operator and leads to serious accidents. The proposed system would navigate that to approach to the target tree selected by the operator autonomously. This navigation was constructed using LiDAR and LiDAR based SLAM which could generate the $3 \mathrm{D}$ map and self-localization. We demonstrated in the experimental field and verification of the navigation system.

\section{Tree harvesting scenario}

Fig. 1 shows the scenario of harvesting process assumed in this study. The details of the scenario are as follows.

1) Selection of a target trees to be felled by the operator (Fig. 1(A)).

2) Approaching the tree by autonomous movement (Fig. 1(B)).

3) Felling of the tree (Fig. 1(B)).

4) Skidding of the tree by autonomous movement (Fig. $1(\mathrm{C}))$.

In this scenario, the approach and skidding movements are automated by the autonomous moving system, the preparation for the felling and the execution of the sawing are subject to the operator's operation.

Considering a situation where $N$ trees are lined up in a straight line, and the machine fells and skids them from the vicinity of the roadside in order. $T_{A n}[\mathrm{sec}]$ is a time it takes to approach the n-th tree, $T_{S n}[\mathrm{sec}]$ is a time it takes to skid the felled tree to the roadside, define the time $T_{n}$ [sec] for autonomous movement as $T_{n}=T_{A n}+T_{S n}$. Let $L[\mathrm{~m}]$ be the distance of neighborhood tree form the roadside, $l[\mathrm{~m}]$ be the interval of trees, and $v[\mathrm{~m} / \mathrm{s}]$ is the average movement velocity of the machine. $T_{A n}$ and $T_{S n}$ is approximately equal and can be estimated by Eq. (1).

$$
T_{A n}=T_{S n}=\left\{\frac{L+(n-1) l}{v}\right\}
$$

The sum of the time to prepare for felling and to execute of felling is defined as $T_{F}$ [sec], the sum of the time to

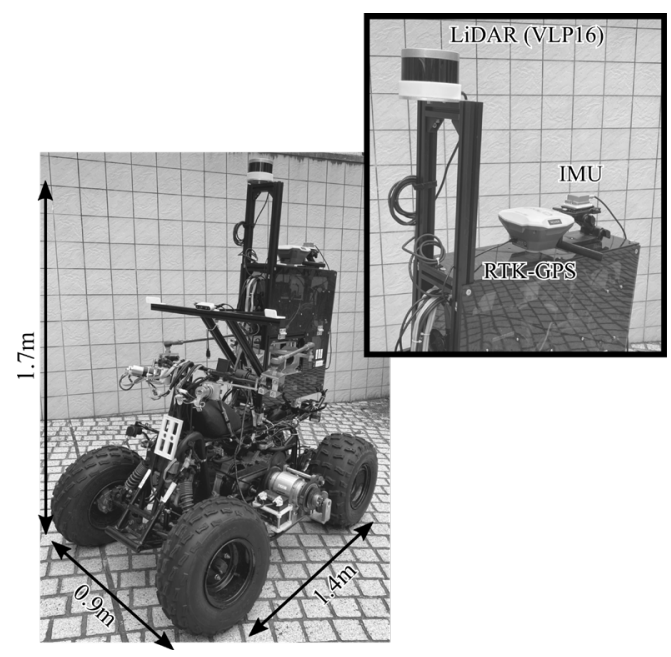

Fig. 2. Forestry robot "SOMA"

select the target tree, it takes for the felled tree to be lowered to an unobstructed position and for the operator to carried-out the operation in $\tau$ [sec]. The total time $T_{N}$ [sec] required to complete the harvesting of the whole trees can be estimated by Eq. (2). Here, $T_{F}$ and $\tau$ are assumed to be the equal for all trees.

$$
\begin{aligned}
T_{N} & =\sum_{n=1}^{N} T n+N T_{F}+N \tau \\
& =2 \sum_{n=1}^{N}\left\{\frac{L+(n-1) l}{v}\right\}+N\left(T_{F}+\tau\right)
\end{aligned}
$$

The first term in Eq. (2) is the total time involved in moving, with the progression of the work, the ratio to the total work time $\sum_{n=1}^{N} T_{n} / T_{N}$ will increase.

\section{System structure}

In this study, we developed autonomous ground vehicle "SOMA" shown in Fig. 2. SOMA is based on an AllTerrain Vehicle (ATV) that is robust designed to movement on rough terrain. Fig. 3 shows the system structure that also includes the operation station. The surrounding trees are observed using LiDAR (Velodyne, VLP-16). The robot pose is measured by IMU (Xsense, MTi-30). In this system, the robot was navigated by LiDAR based SLAM which combined IMU's output.

\subsection{HDL Graph SLAM}

The HDL Graph SLAM was developed for the generation of 3D map and self-localization using 


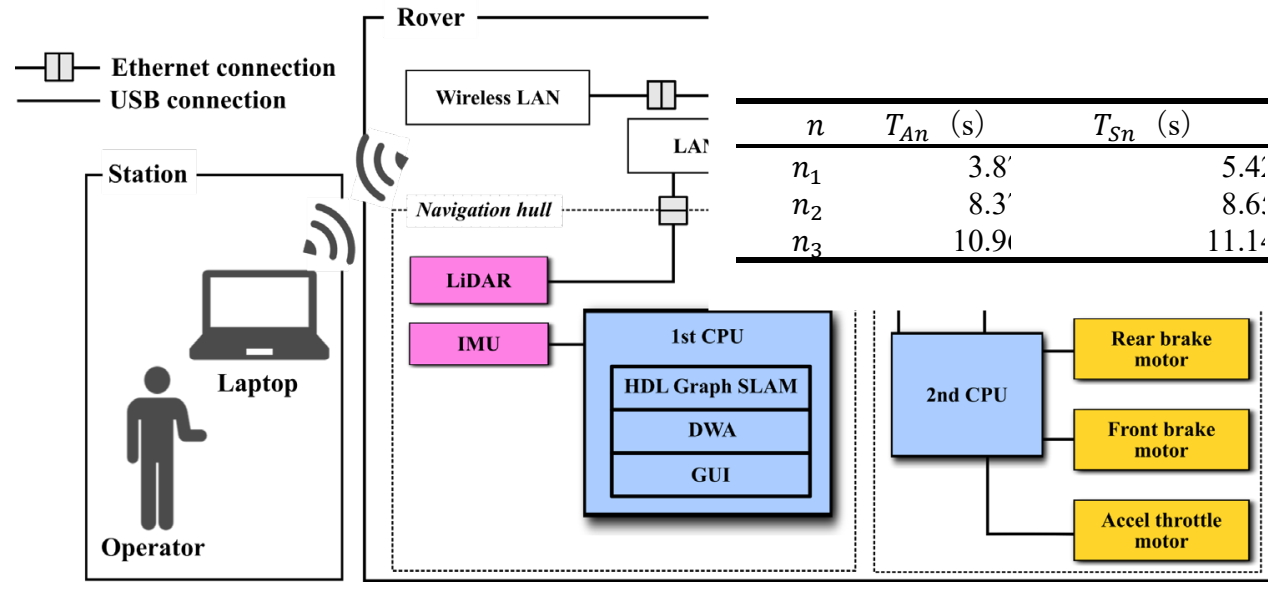

Fig. 4. System structure

LiDAR[1]. It is the SLAM that integrates NDT scanmatching[2] using point clouds obtained from LiDAR and the graph optimization.

\section{2. $D W A$}

The motion plans to approach the target tree is performed by Dynamic Window Approach (DWA) algorithms[3]. The operator could select the target tree of harvesting, the position $(x, y)$ of it's the point will be global target position. The control input (liner velocity, and angular velocity) would be determined by DWA.

\section{Verification experiment}

We demonstrated the automatic harvesting process to verify the effectiveness of the proposed system in the experimental field. Fig. 4 shows the setting of the initial situation of experiment. The 9 imitation trees were placement equally interval. Approaching and skidding performed for the second row. In this experiment, the robot approached the target autonomously and transitioned to the waiting state for the felling command. After an appropriate amount of time, the robot returned to its initial position by issuing a discharge command. Table 1. Experimental Results the time spent on each element work. 250.8 seconds to complete the process of felling in three targets. The ratio of autonomous moving time to total work time was $9.3,14.6$ and $19.2 \%$ as the work progressed. This suggests that this system is effective in operations such as row thinning, where the trees are felled and skidded in sequence. It was confirmed that the round-trip movement was achieved as expected, and the ratio of autonomous moving time in working time increased.

\section{Conclusions}

In this study, the navigation system based on LiDAR and SLAM was developed to reduce the burden of workers in the harvesting process. The future work includes evaluation of the approaching accuracy and motion planning to achieve a stable and accurate approach and return to the roadside.

\section{References}

1. K. Koide, J. Miura, and E. Menegatti, A portable threedimensional LIDAR-based system for long-term and wide-area people behavior measurement, Int. J. Adv. Robot. Syst., 16(2), 2019.

2. P. Biber and W. Strasser, The normal distributions transform: a new approach to laser scan matching, Proceedings 2003 IEEE/RSJ International Conference on Intelligent Robots and Systems (IROS 2003), 2013, 3, pp. 2743-2748.

3. D. Fox, W. Burgard, and S. Thrun, The dynamic window approach to collision avoidance, IEEE Robot. Autom. Mag., 4(1), 1997, pp. 23-33. 